\title{
Concomitant early avascular necrosis of the femoral head and acute bacterial arthritis by enteric Gram- negative bacilli in four oncologic patients
}

Tien Jin $\underline{\operatorname{Tan}}^{1}$, MBBS, FRCR, Suan Cheng $\underline{\operatorname{Tan}^{1}}$, MBBS, FRCR

\begin{abstract}
We present four cases of concomitant early (modified Ficat-Arlet stage I) avascular necrosis of the femoral head and acute bacterial arthritis of the hip joint by Gram-negative enteric bacilli. This was found in immunosuppressed oncologic patients whose clinical presentations and radiological findings were not entirely specific for joint sepsis. It is important to recognise the coexistence of these two pathologies, so as to avoid a delay in diagnosis and prevent significant morbidity and mortality.
\end{abstract}

Keywords: avascular necrosis, hip, magnetic resonance imaging, oncology, septic arthritis

\section{INTRODUCTION}

Although avascular necrosis (AVN) is not generally recognised as a risk factor for acute bacterial arthritis, ${ }^{(1,2)}$ the relationship between the two is clinically significant, as it may be difficult to distinguish the two pathologies based on clinical history and physical examination findings. ${ }^{(3)} \mathrm{A}$ high index of suspicion is needed in order to avoid a delay in the diagnosis and treatment of an acutely infected joint, which can potentially result in significant morbidity and mortality. ${ }^{(3,4)}$ We report a series of four oncologic patients with concomitant occurrence of AVN (modified Ficat-Arlet ${ }^{(5)}$ stage I; Table I) of the femoral head and acute bacterial arthritis of the hip joint by enteric Gram-negative bacilli. Table II lists the pertinent details of all four patients.

\section{CASE REPORTS}

\section{Case 1}

A 23-year-old Arabic woman, with stage IIA lymphocytepredominant Hodgkin's lymphoma on long-term prednisolone for presumed bleomycin-induced pulmonary toxicity, presented with a two-day history of low-grade fever, dysuria and left hip pain. Isolates of Pseudomonas aeruginosa and Salmonella (S.) enteritidis from the patient's urine and blood cultures, respectively, were sensitive to intravenous ceftriaxone, which had been started empirically. Conventional radiographs of the left hip were normal. However, fat-saturated T2-weighted magnetic resonance (MR) images revealed a small left hip joint effusion and the double line sign. The latter consisted of a high signal intensity serpentine line (representing hyperaemic granulation tissue) inside a parallel rim of decreased signal intensity (representing sclerotic bone) in the anterior aspect of the left femoral head, which was characteristic of $A V N^{(6)}$ (Fig. 1).
Table I. Modified Ficat-Arlet ${ }^{(5)}$ classification of avascular necrosis of the femoral head.

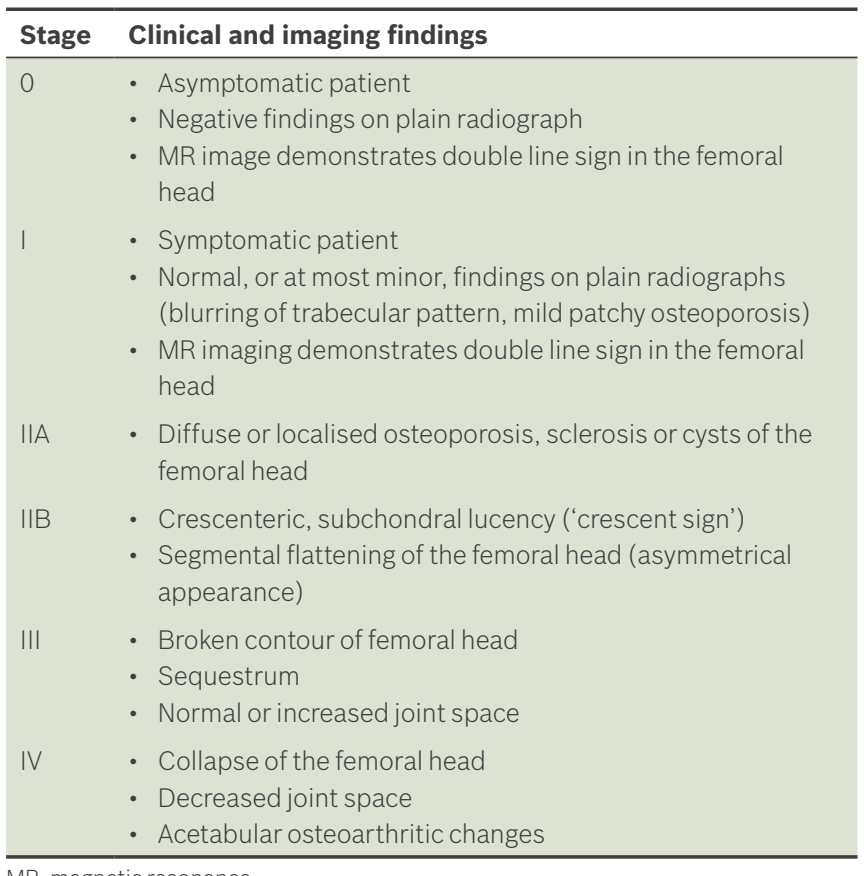

Mild left hip joint synovial thickening and enhancement was observed in contrast-enhanced fat-saturated T1-weighted MR images (Fig. 2). Computed tomography (CT)-guided needle aspiration of the left hip yielded purulent fluid, but cultures were negative. Left hip arthrotomy, washout and synovectomy were performed due to a lack of clinical improvement in the patient despite antibiotic therapy. There was rapid resolution of the patient's fever and left hip pain following surgery. By the time of her discharge from hospital, she was symptom-free and ambulating independently after completing four weeks of intravenous antibiotics.

${ }^{1}$ Department of Diagnostic Radiology, Singapore General Hospital, Singapore

Correspondence: Dr Tan Tien Jin, Associate Consultant, Department of Diagnostic Radiology, Changi General Hospital, 2 Simei Street 3 , Singapore 529889. tien_jin_tan@cgh.com.sg 
Table II. Clinical and radiological features of the present cases.

\begin{tabular}{|c|c|c|c|c|c|c|c|}
\hline Case & Gender & Age (yrs) & $\begin{array}{l}\text { Underlying } \\
\text { malignancy }\end{array}$ & $\begin{array}{l}\text { Clinical } \\
\text { presentation }\end{array}$ & $\begin{array}{l}\text { Radiograph } \\
\text { findings }\end{array}$ & MR imaging findings & Isolated organism \\
\hline 1 & Female & 23 & $\begin{array}{l}\text { Stage IIA } \\
\text { Hodgkin's } \\
\text { lymphoma }\end{array}$ & $\begin{array}{l}\text { Left hip pain, } \\
\text { low-grade fever, } \\
\text { dysuria }\end{array}$ & $\begin{array}{l}\text { Hip radiographs } \\
\text { negative }\end{array}$ & $\begin{array}{l}\text { Modified Ficat-Arlet }{ }^{(5)} \text { stage I } \\
\text { AVN of the left femoral head; } \\
\text { left hip joint effusion and } \\
\text { synovitis }\end{array}$ & $\begin{array}{l}\text { Salmonella enteritidis from } \\
\text { blood culture, } \\
\text { Pseudomonas aeruginosa from } \\
\text { urine culture }\end{array}$ \\
\hline 2 & Male & 55 & $\begin{array}{l}\text { Stage II multiple } \\
\text { myeloma }\end{array}$ & $\begin{array}{l}\text { Right hip pain, } \\
\text { low-grade fever }\end{array}$ & $\begin{array}{l}\text { Hip radiographs } \\
\text { negative }\end{array}$ & $\begin{array}{l}\text { Modified Ficat-Arlet(5) stage I } \\
\text { AVN of the right femoral head; } \\
\text { right hip joint effusion, synovitis } \\
\text { and iliopsoas bursitis }\end{array}$ & $\begin{array}{l}\text { Salmonella enteritidis from right } \\
\text { iliopsoas bursal aspirate }\end{array}$ \\
\hline 3 & Female & 34 & $\begin{array}{l}\text { Acute myeloid } \\
\text { leukaemia }\end{array}$ & $\begin{array}{l}\text { Left hip pain, } \\
\text { low-grade fever }\end{array}$ & $\begin{array}{l}\text { Hip radiographs } \\
\text { negative, } \\
\text { chest radiograph } \\
\text { showed presence } \\
\text { of pneumonia }\end{array}$ & $\begin{array}{l}\text { Modified Ficat-Arlet }{ }^{(5)} \text { stage I } \\
\text { AVN of both femoral heads; } \\
\text { left hip joint effusion and } \\
\text { synovitis }\end{array}$ & $\begin{array}{l}\text { Escherichia coli from blood } \\
\text { culture and left hip aspirate }\end{array}$ \\
\hline 4 & Male & 60 & $\begin{array}{l}\text { Stage IV lung } \\
\text { adenocarcinoma }\end{array}$ & Right hip pain & $\begin{array}{l}\text { Hip radiographs } \\
\text { negative }\end{array}$ & $\begin{array}{l}\text { Modified Ficat-Arlet }{ }^{(5)} \text { stage I } \\
\text { AVN of the right femoral head; } \\
\text { right hip joint effusion and } \\
\text { synovitis }\end{array}$ & $\begin{array}{l}\text { Salmonella enteritidis from right } \\
\text { hip aspirate }\end{array}$ \\
\hline
\end{tabular}

\section{Case 2}

A 55-year-old Arabic man, who was being treated with bortezomib and dexamethasone for stage II multiple myeloma, presented with a two-day history of right hip pain. Conventional radiographs of the right hip were normal. Characteristic of AVN, $M R$ imaging revealed the presence of the double line sign in the right femoral head. MR imaging also revealed communication between a right iliopsoas bursitis and a moderate right hip joint effusion (Fig. 3). There was associated marked synovial thickening and enhancement in the right hip joint and right iliopsoas bursa (Fig. 4). The patient suffered from mild pyrexia and had an elevated C-reactive protein (CRP) level of $176 \mathrm{mg} / \mathrm{L}$, prompting a search for the source of sepsis. $S$. enteritidis was eventually isolated from the bursal fluid at the third episode of CT-guided needle aspiration. The patient was started and later maintained on intravenous ceftriaxone, as guided by culture and sensitivity results. A decision was made to proceed with right hip arthrotomy, washout and synovectomy after obtaining a positive culture. $S$. enteritidis was isolated from cultures of fluid obtained from the infected right hip joint at surgery. The patient's right hip pain subsequently resolved, and he was ambulatory with the aid of a walking stick upon discharge, after completing four weeks of intravenous antibiotic therapy.

\section{Case 3}

A 34-year-old Chinese woman was admitted electively for salvage chemotherapy with idarubucin, cytarabine and sorafenib, following the diagnosis of relapsed acute myeloid leukaemia (AML). She had been maintained on prednisolone following an allogenic bone marrow transplant performed a year earlier for AML. She developed low-grade fever and left hip pain on Day 3 of admission. Chest radiography performed as part of a septic workup revealed the presence of bilateral pulmonary infiltrates, in keeping with pneumonia. Conventional radiographs of the left hip were normal. The patient was found to have Escherichia (E.) coli bacteraemia and was thus started on intravenous

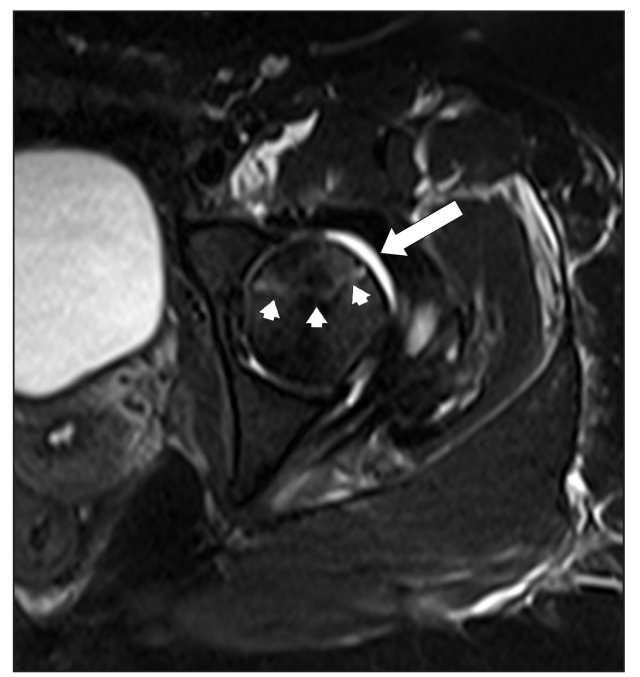

Fig. 1 Case 1: Axial fat-saturated T2-W MR image of the left hip shows the double line sign (arrowheads) in the anterior aspect of the left femoral head, which is characteristic of avascular necrosis. Note the small left hip joint effusion (arrow).

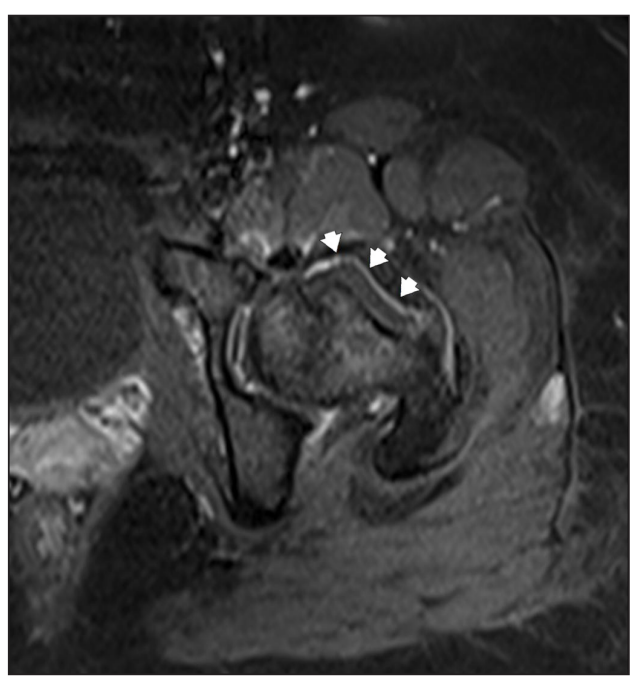

Fig. 2 Case 1: Axial contrast-enhanced, fat-saturated T1-W MR image of the left hip shows mild synovial thickening and enhancement (arrowheads). 


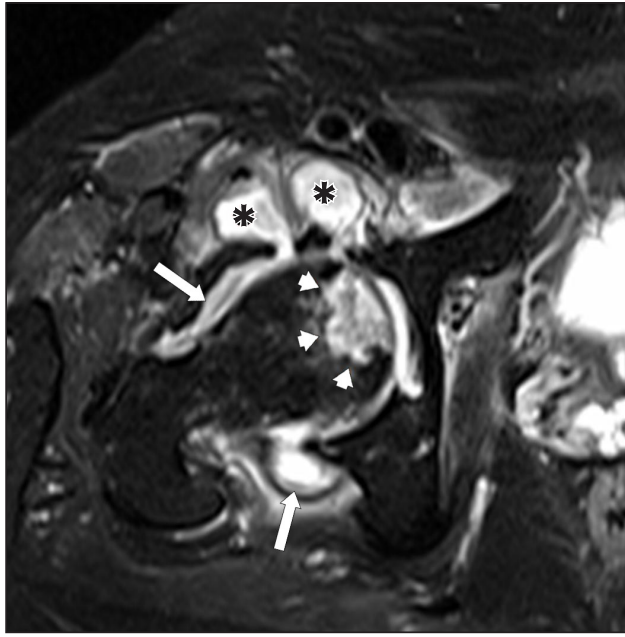

Fig. 3 Case 2: Axial fat-saturated T2-W MR image of the right hip shows the double line sign in the right femoral head, which is characteristic of avascular necrosis (arrowheads), and a right iliopsoas bursitis (*) communicating with a moderate right hip joint effusion (arrows).

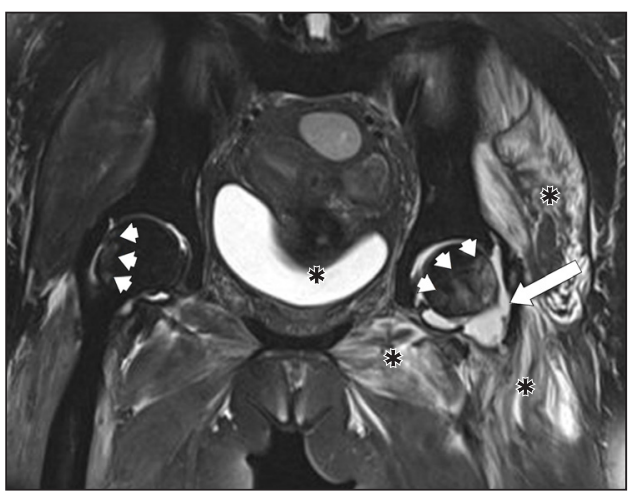

Fig. 5 Case 3: Coronal fat-saturated T2-W MR image of both hip joints shows the double line sign (arrowheads), which is characteristic of avascular necrosis, in both femoral heads, as well as a moderate left hip joint effusion (arrow) and surrounding myositis (*).

levofloxacin. She was subsequently deemed to have recovered sufficiently from pneumonia and began receiving salvage chemotherapy. MR imaging of her left hip performed for persistent left hip pain demonstrated the double line sign, which was characteristic of AVN, in both femoral heads, as well as a moderate left hip joint effusion and surrounding myositis (Fig. 5). There was also synovial thickening and enhancement in the patient's left hip joint (Fig. 6). Ultrasonography-guided needle aspiration of the left hip joint was performed, which yielded purulent fluid. The patient's antibiotic regimen was revised to intravenous ertapenem after multidrug-resistant $E$. coli was isolated from the joint aspirate. She subsequently underwent left hip arthrotomy, washout and synovectomy. She remained afebrile and her left hip pain rapidly subsided after surgery. She was ambulatory with the assistance of a walking frame by the time of her discharge, after completion of four weeks of intravenous antibiotics.

\section{Case 4}

A 60-year-old Chinese man with stage IV lung adenocarcinoma presented with a three-day history of right hip pain. He had

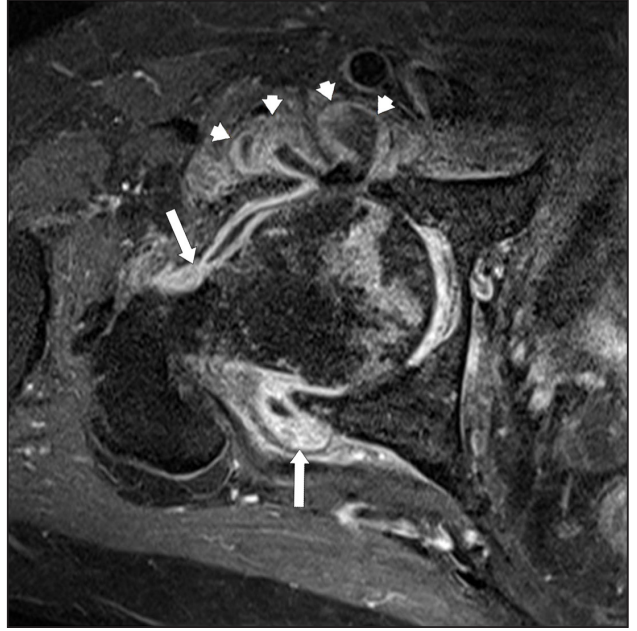

Fig. 4 Case 2: Axial contrast-enhanced, fat-saturated T1-W MR image of the right hip shows marked synovial thickening and enhancement within the right hip joint (arrows) and right iliopsoas bursa (arrowheads).

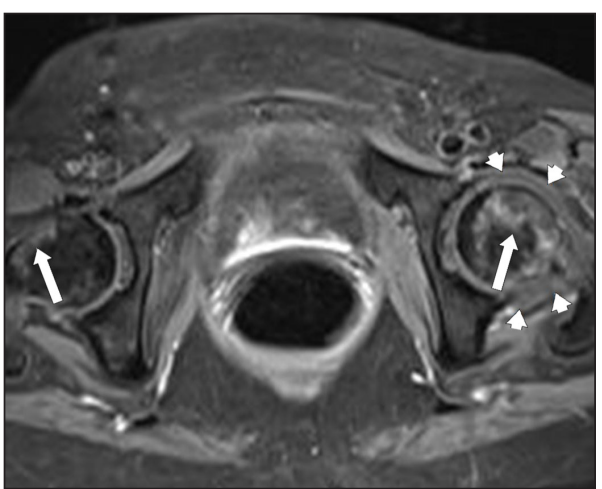

Fig. 6 Case 3: Axial contrast-enhanced, fat-saturated T1-W MR image of both hip joints shows the presence of avascular necrosis in both femoral heads (arrows). Synovial thickening and enhancement in the left hip joint (arrowheads) is also present.

previously undergone left upper lobectomy to remove a primary lung tumour, as well as stereotactic radiosurgery and whole brain radiotherapy for cerebral metastases. He had also been started on a chemotherapy regimen that included a combination of gemcitabine, carboplatin and gefitinib for three weeks, as well as dexamethasone for two months prior to his presentation. Conventional radiographs of the right hip were unremarkable. MR imaging of the right hip revealed AVN of the right femoral head, evidenced by the double line sign, as well as synovial thickening and enhancement associated with a small right hip joint effusion. These imaging appearances are similar to those found in Case 1. Prompted by persistent symptoms and a raised CRP level of $326 \mathrm{mg} / \mathrm{L}$, ultrasonography-guided needle aspiration of the patient's right hip joint was performed, yielding purulent fluid. A diagnosis of septic arthritis due to $S$. enteritidis was subsequently established. The patient's treatment (he had initially been treated empirically with intravenous ceftriaxone) was switched to intravenous ciprofloxacin based on culture and sensitivity results. Right hip arthrotomy, washout and synovectomy were also performed after the result of a positive culture. There was significant improvement and eventual 
resolution of the patient's symptoms following surgery and completion of four weeks of intravenous therapy. He was asymptomatic and fully ambulatory by the time of his discharge from hospital.

\section{DISCUSSION}

Establishing a diagnosis of acute bacterial arthritis presents no difficulty in a patient with typical symptoms of fever associated with a warm, swollen and exquisitely painful joint. However, its clinical presentation may be nonspecific when the onset of septic arthritis is insidious or if the host's response to infection is impaired. In such cases of impaired response to infection, symptoms of systemic infection such as fever may only be detected in up to $60 \%$ of cases. ${ }^{(4)}$ Low-grade fever recorded in Cases 1 and 3 was deemed secondary to concomitant urinary tract infection and pneumonia, respectively, rather than joint sepsis. Based on the protracted course of corticosteroids and MR imaging findings, an initial diagnosis of AVN of the femoral heads was made in all four cases. However, septic arthritis could not be excluded in these immunocompromised patients with low-grade pyrexia and/ or raised serum inflammatory markers. The association between $\mathrm{AVN}$ and acute bacterial arthritis has been reported, although only infrequently in cases of systemic lupus erythematosus, ${ }^{(7,8)}$ organ transplant, ${ }^{(8,9)}$ sickle-cell disease, ${ }^{(10,11)}$ as well as in chronic alcoholism and intravenous drug abuse. ${ }^{(12)}$ We found no prior reference to oncologic patients in the literature.

The most frequent causative organisms of nongonococcal acute bacterial arthritis are Gram-positive cocci, particularly Staphylococcus aureus. However, both E. coli and S. enteritidis are enteric, anaerobic, Gram-negative bacilli that, being usually encountered in the clinical setting of intravenous drug abuse or major immunodeficiency, are uncommon causative organisms of acute bacterial arthritis. ${ }^{(1,2)}$ The number of reported cases in the literature of $S$. enteritidis causing joint sepsis remains small, with most patients having either a predisposing systemic or joint disease. ${ }^{(13)}$ Bacterial translocation from the gastrointestinal tract is promoted by deficiencies in the host's immune defences. ${ }^{(14)}$ This is the presumed mechanism that resulted in bacteraemia and haematogenous seeding of the infected hip joints in our patients. It is postulated that the predilection for establishment of septic arthritis within the hip joints in our patients was secondary to the presence of a hypoxic environment in the setting of concomitant AVN. Such an environment may have been conducive for the growth of anaerobic microbes, such as those isolated in our case series.

MR imaging is well established as the imaging modality of choice for the investigation of early AVN of the femoral head and asymptomatic contralateral disease. ${ }^{(6)}$ Accordingly, MR imaging proved more useful than conventional radiography in establishing the diagnosis of early AVN in our patients. Additionally, MR imaging findings of joint effusion, and synovial thickening and enhancement supported the suspicion of concomitant septic arthritis, prompting needle aspiration, which eventually led to the diagnosis of acute bacterial arthritis. Arthrocentesis is the best diagnostic tool available for the detection of bacterial arthritis. ${ }^{(3)}$ In three of our cases, synovial fluid analysis was critical in establishing the diagnosis of acute bacterial arthritis and identifying the causative organism. With respect to Case 1, the inability to culture any organism from joint fluid was not unexpected, as the patient was already on intravenous antibiotics when aspiration of her hip joint was undertaken. The diagnosis of septic arthritis in this case was thus based on positive blood cultures and the presence of purulent fluid in the hip aspirate.

General consensus states that appropriate treatment should be expedited once acute bacterial arthritis is suspected, in view of its rapidly destructive nature. ${ }^{(1,2,4)}$ Broad-spectrum antibiotics are generally recommended until culture results are available to guide further antibiotic therapy. In addition to antimicrobial treatment, removal of intra-articular pus via needle aspiration, arthroscopy or open surgery is usually necessary. Our patients were aggressively treated with intravenous antibiotics and surgery once the diagnosis of acute bacterial arthritis was established, achieving good clinical response. Our series of immunocompromised oncologic patients presented with acute hip pain and were found to have early stage AVN of the femoral head on MR imaging. However, their symptoms were not simply attributed to AVN, as there was strong suspicion of concomitant septic arthritis in view of clinical findings of pyrexia, raised inflammatory markers (such as CRP level) and MR imaging findings of joint effusion and synovitis. Prompt arthrocentesis, which guided definitive treatment, was performed in all of the cases.

\section{ACKNOWLEDGEMENT}

The authors would like to thank Dr David Tai Wai Meng, Associate Consultant Oncologist at the National Cancer Centre Singapore, for the invaluable assistance rendered in the proofreading of the manuscript.

\section{REFERENCES}

1. Ross JJ. Septic arthritis. Infect Dis Clin North Am 2005; 19:799-817.

2. García-De La Torre I. Advances in the management of septic arthritis. Infect Dis Clin North Am 2006; 20:773-88.

3. Margaretten ME, Kohlwes J, Moore D, Bent S. Does this adult patient have septic arthritis? JAMA 2007; 297:1478-88.

4. Matthews CJ, Weston VC, Jones A, Field M, Coakley G. Bacterial septic arthritis in adults. Lancet 2010; 375:846-55

5. Mont MA, Hungerford DS. Non-traumatic avascular necrosis of the femoral head. J Bone Joint Surg Am 1995; 77:459-74.

6. Mitchell DG, Rao VM, Dalinka MK, et al. Femoral head avascular necrosis: correlation of MR imaging, radiographic staging, radionucleotide imaging, and clinical findings. Radiology 1987; 162:709-15.

7. Markov G, Dobro J, Shankman S, Belmont HM. Enterococcal arthritis with avascular necrosis in a lupus patient. Br J Rheumatol 1996; 35:595-7.

8. Habermann ET, Friedenthal RB. Septic arthritis associated with avascular necrosis of the femoral head. Clin Orthop Relat Res 1978; (134):325-31.

9. Ostrum RF. Nocardia septic arthritis of the hip with associated avascular necrosis. A case report. Clin Orthop Relat Res 1993; (288):282-6. 
10. Hernigou P, Daltro G, Flouzat-Lachaniette $\mathrm{CH}$, Roussignol X, Poignard A. Septic arthritis in adults with sickle cell disease is associated with osteomyelitis or osteonecrosis. Clin Orthop Relat Res 2010; 468:1676-81.

11. Phillips FM, Pottenger LA. Acute septic arthritis in chronic osteonecrosis of the hip. J Rheumatol 1988; 15:1713-6.

12. Nuovo MA, Sissons HA, Zuckerman JD. Case report 662. Bilateral avascular necrosis of femur, with supervening suppurative arthritis of right hip. Skeletal Radiol 1991; 20:217-21.

13. Dineen PF, Harty JA, Dolan AM. Septic arthritis of the ankle due to Salmonella enteritidis. Foot Ankle Surg 2011; 17:e23-4.

14. Berg RD. Bacterial translocation from the gastrointestinal tract. Trends Microbiol 1995; 3:149-54. 